\title{
OPTIMAL CONTROL ANALYSIS OF HIV/AIDS TRANSMISSION IN THE PRESENCE OF CAREFUL AND CARELESS INDIVIDUALS
}

\author{
BABA SEIDU ${ }^{1, *}$, OLUWOLE D. MAKINDE $^{2}$, CHRISOPHER S. BORNAA $^{3}$ \\ ${ }^{1}$ Mathematics Department, Faculty of Mathematical Sciences, C. K. Tedam University of Technology and \\ Applied Sciences, Navrongo, Ghana \\ ${ }^{2}$ Faculty of Military Science, Stellenbosch University, South Africa \\ ${ }^{3}$ Department of Science and Mathematics Education, C. K. Tedam University of Technology and Applied \\ Sciences, Ghana \\ *Corresponding author: bseidu@cktutas.edu.gh \\ Received Feb. 21, 2021
}

\begin{abstract}
AвstRact. This paper proposes a nonlinear deterministic ordinary differential equations model that describes the dynamics of HIV/AIDS transmission in the presence of careful and careless Susceptibles and Infectives. It is shown that when $\mathcal{R}_{0}<1$, the model exhibits a locally asymptotically stable disease-free equilibrium, and global stability is guaranteed only under some conditions. Incremental-cost-effectiveness ratio analysis of results from optimal control analysis of the model showed that the best strategy that should be implemented in the fight against the spread of HIV/AIDS is one that involves prevention and behavior change.
\end{abstract}

2010 Mathematics Subject Classification. 92D30; 37N25; 34D20; 92B05; 92D25.

Key words and phrases. human immunodeficiency virus (HIV); basic reproduction number; bifurcation; optimal control analysis.

\section{INTRODUCTION}

The Human Immunodeficiency Virus (HIV) is virus that attacks the white blood cells that are responsible for fighting pathogen invasion. The viral attack leads to reduction in the CD4+ cell count. When the CD4+ cell count goes below 500, the body loses its ability to fight against diseases and the body is said to have Acquired Immunodeficiency Syndrome (AIDS).

Mathematical modelling has played a very important role in increasing our understanding of

DOI: 10.28924/APJM/8-15

(2021 Asia Pacific Journal of Mathematics 
dynamics of infectious diseases. Several models have been proposed and analyzed by many researchers to describe the pathogenesis of infectious diseases (See $[8,11-13]$ and others). Other authors have proposed models to study the impact of some social and demographic factors that effect the spread of some diseases (See $[1,3,4,9,15]$ among others). In recent years, there has been a tremendous increase in attempts to develop and solve optimal control problems that are aimed at providing optimal strategies of controlling disease spread. This paper is one of such models that seek to use optimal control theory in the analysis of dynamics of HIV / AIDS. In this paper, we propose a model that describes the dynamics of HIV in the presence of careful and careless infectives and Susceptibles. Our interest is to find out how varying attitudes towards sex affects the spread of the disease and subsequently determine optimal strategies that can used to combat the spread of the disease.

The remainder of the paper is organized as follows. The next Section presents the derivation of the model, while in section 3, the basic properties of the model are presented. Further analysis in the form of bifurcation is carried out in section 4 . In section 5 , we extend the model into an optimal control problem, which is analyzed qualitatively. In section 6, numerical simulation of the model is carried out. The conclusions drawn from the paper are presented in section 7.

\section{Model Formulation}

The model considers a homogeneously mixed human population of total size $N(t)$ at time $t$ that is sub-divided into five mutually exclusive classes of Careful Susceptibles, $S_{1}(t)$, Careless Susceptibles, $S_{2}(t)$, Careful Infectives, $I_{1}(t)$, Careless Infectives, $I_{2}(t)$ and full-blown AIDS patients, $A(t)$. Thus the total population is given by:

$$
N(t)=S_{1}(t)+S_{2}(t)+I_{1}(t)+I_{2}(t)+A(t)
$$

Careful individuals are people who exhibit characteristics that lead to reduced chances of contracting or transmitting HIV. Careful behavior include abstinence, faithfulness towards sexual partners and proper/regular use of condom among others. Careless individuals on the other hand are people whose behavior increases their chances of contracting or transmitting HIV. Let $Q$ be the rate of immigration into the population with $m_{1}, m_{2}$ and $m_{3}$ being the fractions of immigrants who are Careless Susceptibles, Careful Infectives, and Careless Infectives respectively. The fraction of immigrants who are Careful Susceptibles is then $1-m_{1}-m_{2}-m_{3}$. Assuming that there is a policy of education and/or counseling that seeks to change the behavior of careless individuals so that the become careful. Let $b_{s}$ and $b_{i}$ be the rate at which 
careless Susceptibles and Infectives become careful Susceptibles and Infectives respectively. For the present paper, we consider a standard incidence rate with force of infection given by $\lambda=\frac{c \beta\left(1-u_{1}\right)\left(I_{1}+p_{1} I_{2}+p_{2} A\right)}{N}$, where $c, \beta, p_{1}, p_{2}$ and $p_{3}$ are as defined in Table 1 . We assume that careful and careless Susceptibles, when infected through contact with infectives, move to careful and careless Infectives respectively. That is, careful Susceptibles become careful Infectives after infection, likewise the careless individuals. We consider also that infectives progress into the full-blown AIDS class at rate $\delta$. The parameter $\delta$ could also be thought of as the failure rate of treatment of infectives. We incorporate time dependent control $u_{i}(t), i=1,2,3$, where $u_{1}(t)$ is the control variable that represents a successful use of condom by Susceptibles to prevent infection; $u_{2}(t)$ is successful counseling of careless individuals so that they become careful; and $u_{3}(t)$ represents a successful implementation of anti-retro-viral therapy.

The dynamics of the spread of HIV / AIDS is thus given by the following set of differential equations.

$$
\left\{\begin{array}{l}
\frac{\mathrm{d} S_{1}}{\mathrm{~d} t}=\left(1-m_{1}-m_{2}-m_{3}\right) Q+u_{2} b_{s} S_{2}-(\lambda+\mu) S_{1} \\
\frac{\mathrm{d} S_{2}}{\mathrm{~d} t}=m_{1} Q-\left(p_{3} \lambda+u_{2} b_{s}+\mu\right) S_{2} \\
\frac{\mathrm{d} I_{1}}{\mathrm{~d} t}=m_{2} Q+\lambda S_{1}+b_{i} u_{2} I_{2}-\left(\delta\left(1-u_{3}\right)+\mu\right) I_{1} \\
\frac{\mathrm{d} I_{2}}{\mathrm{~d} t}=m_{3} Q+p_{3} \lambda S_{2}-\left(b_{i} u_{2}+\eta \delta\left(1-u_{3}\right)+\mu\right) I_{2} \\
\frac{\mathrm{d} A}{\mathrm{~d} t}=\delta\left(1-u_{3}\right)\left(I_{1}+\eta I_{2}\right)-(\psi+\mu) A
\end{array}\right.
$$

For simplification, we shall use the representations:

$$
k_{1}=p_{3} \lambda+u_{2} b_{s}+\mu, k_{2}=\delta\left(1-u_{3}\right)+\mu, k_{3}=b_{i} u_{2}+\eta \delta\left(1-u_{3}\right)+\mu \text { and } k_{4}=\psi+\mu
$$

All parameters are considered to be non-negative in the subsequent discussions.

\section{Basic Properties of the Model}

This section presents some basic properties of the model under discussion.

3.1. Feasible Region. One of the very important properties of epidemic models is that they be epidemiologically and biologically reasonable. That is, the model should produce reasonable results. It is easy to prove that if the initial conditions are non-negative, then all solutions of the model will remain non-negative for all future time $t>0$. The following theorem is thus in order.

Theorem 3.1. If $S_{1}(0), S_{2}(0), I_{1}(0), I_{2}(0)$ and $A(0)$ are non-negative, then $S_{1}(t), S_{2}(t), I_{1}(t), I_{2}(t)$ and $A(t)$ are non-negative for all $t>0$. Moreover, $\limsup _{t \rightarrow \infty} N(t) \leq \frac{Q}{\mu}$. 
TABle 1. Description of Parameters used in the Model

\begin{tabular}{|c|l|l|l|}
\hline Parameter & Description & Value $(/ \mathrm{yr})$ & Ref. \\
\hline $\mathrm{Q}$ & Rate of immigration into Population & 100 & \\
\hline$m_{1}, m_{2}, m_{3}$ & Fraction of immigrants into $S_{2}, I_{1}$ and $I_{2}$ classes respectively & $0.30,0.20,0.10$ & \\
\hline$b_{s}$ & Rate of positive behavioral change of careless Susceptibles & 0.60 & \\
\hline$b_{i}$ & Rate of positive behavioral change of careless Infectives & 0.40 & \\
\hline$\delta$ & Rate of progression of untreated infectives into AIDS class & 0.1 & {$[1]$} \\
\hline$p_{1}, p_{2}$ & Modification parameter due to increased infectivity of $I_{2}$ and $A$ & $1.50,1.70$ resp. & \\
\hline$p_{3}$ & Modification parameter due to increased Susceptibility of $S_{2}$ & 2.00 & \\
\hline$\eta>1$ & Modification parameter due to increased progression of $I_{2}$ into $A$ & 1.40 & \\
\hline$\mu$ & Natural death rate of humans & 0.02 & {$[1]$} \\
\hline$\psi$ & AIDS-induced death rate of humans & 1.00 & {$[1]$} \\
\hline
\end{tabular}

Thus, we shall analyze the model inside the feasible region,

$$
\Omega=\left\{\left(S_{1}, S_{2}, I_{1}, I_{2}, A\right) \in \mathbb{R}_{+}^{5} \mid S_{1}+S_{2}+I_{1}+I_{2}+A \leq \frac{Q}{\mu}\right\} .
$$

The feasible region, $\Omega$ can be proved to be positively invariant. The model is therefore mathematically well-posed [14]. Hence, we can be sufficiently studied in the region $\Omega$.

3.2. Local Stability of the DFE. The DFE of the model exhibits a disease-free equilibrium given by $E_{0}=\left(\left(1-\frac{m_{1} \mu}{b_{s} u_{2}+\mu}\right) \frac{Q}{\mu}, \frac{m_{1} Q}{b_{s} u_{2}+\mu}, 0,0,0\right)$.

Using the method of Driessche and Watmough [10] the basic reproduction number, $\mathcal{R}_{0}$, is given by:

$\mathcal{R}_{0}=\frac{c \beta\left[\left(p_{2} \delta\left(1-u_{3}\right)+k_{4}\right)\left(k_{3} k_{1}-\mu m_{1} k_{3}+\mu b_{i} u_{2} m_{1} p_{3}\right)+\mu p_{3} m_{1}\left(p_{2} \delta \eta\left(1-u_{3}\right)+k_{4} p_{1}\right) k_{2}\right]}{k_{1} k_{2} k_{3} k_{4}}$.

Following Theorem 2 of Driessche and Watmough [10], the following theorem is obtained.

Theorem 3.2. The disease-free equilibrium point $E_{0}$ of the model is LAS when $\mathcal{R}_{0}<1$.

3.3. Sensitivity Analysis of $\mathcal{R}_{0}$. To determine the effect of the model parameters on the basic reproduction number, $\mathcal{R}_{0}$, we carry out sensitivity analysis in this section. We use the normalized forward-sensitivity index measures the percentage change in a dependent variable relative to percentage changes in independent variables. Given that $\mathcal{R}_{0}$ depends on, say $x$, then the normalized forward-sensitivity index of $\mathcal{R}_{0}$ with respect to $x$ is defined as follows:

$$
\Upsilon_{x}^{\mathcal{R}_{0}}=\frac{\partial \mathcal{R}_{0}}{\partial x} \times \frac{x}{\mathcal{R}_{0}}
$$


Therefore we have $\Upsilon_{c}^{\mathcal{R}_{0}}=1, \Upsilon_{\beta}^{\mathcal{R}_{0}}=1$, Due to the complex nature of some of the other sensitivity indexes, we used the parameter values in Table 1 to evaluate the indexes and present them (in decreasing order of impact) in Table 2.

TABLE 2. Sensitivity Indexes of $\mathcal{R}_{0}$ relative to Model Parameters

\begin{tabular}{|l|c|c|c|c|c|c|c|c|c|c|c|c|}
\hline Parameter & $\mathrm{c}$ & $\beta$ & $\delta$ & $\mu$ & $p_{2}$ & $\psi$ & $p_{3}$ & $m_{1}$ & $b_{s}$ & $p_{1}$ & $\eta$ & $b_{i}$ \\
\hline Index & 1 & 1 & 0.691 & 0.158 & 0.143 & 0.14 & 0.021 & 0.011 & 0.011 & 0.005 & 0.004 & 0.001 \\
\hline Sign & + & + & - & - & + & - & + & + & - & + & - & - \\
\hline
\end{tabular}

A positive index implies that an increase (decrease) in the parameter will lead to an increase (decrease) in $\mathcal{R}_{0}$ while a negative index implies that an increase (decrease) of the parameter will lead to a decrease (increase) in $\mathcal{R}_{0}$. Therefore from Table 2, a 10\% increase (or decrease) of either $\beta$ or $c$ will lead to a $10 \%$ increase (or decrease) in $\mathcal{R}_{0}$. Therefore, in order to successfully combat the spread of HIV/AIDS, there is the need to reduce the number of sexual partners of infectives, probability of infection, $p_{1}, p_{2}$ and $p_{3}$; and increase rate of progression into full-blown AIDS, natural death rate and AIDS-induced death rate among others.

3.4. Global Stability of the DFE. We employ the technique of Castillo-Chavez, Blower, Driessche, Kirschner and Yakubu [6] to determine the global asymptotic stability of the disease-free equilibrium. By this technique, if $X=\left(S_{1}, S_{2}\right) \in \mathbb{R}^{2}$ and $Z=\left(I_{1}, I_{2}, A\right) \in \mathbb{R}^{3}$ denote the Susceptible and infective sub-populations respectively and the model is rewritten as:

$$
\left\{\begin{array}{l}
\frac{\mathrm{d} X}{\mathrm{~d} t}=F(X, Z) \\
\frac{\mathrm{d} Z}{\mathrm{~d} t}=G(X, Z), G(X, 0)=0
\end{array}\right.
$$

The disease-free equilibrium point is globally asymptotically stable if the following two conditions are satisfied.

H1: For the reduced system $\left.\frac{\mathrm{d} X}{\mathrm{~d} t}\right|_{Z=0}=F(X, 0), X^{*}=E_{0}$ is GAS.

H2: $G(X, Z)=D_{Z} G\left(X^{*}, 0\right) Z-\hat{G}(X, Z), \hat{G}(X, Z) \geq 0 \forall(X, Z) \in \Omega$.

In item $\mathrm{H} 2$ above, $D_{Z} G\left(X^{*}, 0\right)$ is the Jacobian of $G(X, Z)$ evaluated at $\left(X^{*}, 0\right)$.

Thus, we have

$$
F(X, Z)=\left(\begin{array}{c}
\left(1-m_{1}-m_{2}-m_{3}\right) Q+b_{s} u_{2} S_{2}-\left(\frac{c \beta\left(1-u_{1}\right)\left(I_{1}+p_{1} I_{2}+p_{2} A\right)}{S_{1}+S_{2}+I_{1}+I_{2}+A}+\mu\right) S_{1} \\
m_{1} Q-\left(\frac{c p_{3} \beta\left(1-u_{1}\right)\left(I_{1}+p_{1} I_{2}+p_{2} A\right)}{S_{1}+S_{2}+I_{1}+I_{2}+A}+b_{s} u_{2}+\mu\right) S_{2}
\end{array}\right)
$$




$$
G(X, Z)=\left(\begin{array}{c}
m_{2} Q+\frac{c \beta\left(1-u_{1}\right)\left(I_{1}+p_{1} I_{2}+p_{2} A\right) S_{1}}{S_{1}+S_{2}+I_{1}+I_{2}+A}+b_{i} u_{2} I_{2}-\left(\delta\left(1-u_{3}\right)+\mu\right) I_{1} \\
m_{3} Q+\frac{c p_{3} \beta\left(1-u_{1}\right)\left(I_{1}+p_{1} I_{2}+p_{2} A\right) S_{2}}{S_{1}+S_{2}+I_{1}+I_{2}+A}-\left(b_{i} u_{2}+\eta \delta\left(1-u_{3}\right)+\mu\right) I_{2} \\
\delta\left(1-u_{3}\right)\left(I_{1}+\eta I_{2}\right)-(\psi+\mu) A
\end{array}\right)
$$

and hence

$$
G(X, 0)=\left(\begin{array}{c}
m_{2} Q \\
m_{3} Q \\
0
\end{array}\right) \neq 0 .
$$

We note that the theorem in Castillo-Chavez et al. [6] that we seek to employ here can not be used since the requirement that $G(X, Z)$ vanish at $Z=0$ is not met. This condition is only met when there are no infective immigrants. Hence, we consider the special case where there are no infective immigrants (ie. $m_{2}=m_{3}=0$ ). In this case, we redefine $F(X, Z)$ and $G(X, Z)$ as follows:

$$
F(X, Z)=\left.F(X, Z)\right|_{m_{2}=m_{3}=0}, \quad \text { and } \quad G(X, Z)=\left.G(X, Z)\right|_{m_{2}=m_{3}=0}
$$

Clearly from the reduced system

$$
\begin{aligned}
\left.\frac{\mathrm{d} X}{\mathrm{~d} t}\right|_{Z=0}=F(X, 0)=\left(\begin{array}{c}
\left(1-m_{1}\right) Q+b_{s} u_{2} S_{2}-\mu S_{1} \\
m_{1} Q-\left(b_{s} u_{2}+\mu\right) S_{2}
\end{array}\right), \\
S_{1}(t)=\left(S_{2}(0)+S_{1}(0)\right) \mathrm{e}^{-t \mu}+\frac{\left[m_{1} Q-\left(b_{s} u_{2}+\mu\right) S_{2}(0)\right]}{\left(b_{s} u_{2}+\mu\right)} \mathrm{e}^{-\left(b_{s} u_{2}+\mu\right) t}+\frac{\left[\left(1-\mathrm{e}^{-t \mu}\right)\left(b_{s} u_{2}+\mu\right)-\mu m_{1}\right] Q}{\mu\left(b_{s} u_{2}+\mu\right)} \text { and } \\
S_{2}(t)=\frac{m_{1} Q}{b_{s} u_{2}+\mu}+\left(S_{2}(0)-\frac{m_{1} Q}{b_{s} u_{2}+\mu}\right) \mathrm{e}^{-\left(b_{s} u_{2}+\mu\right) t},
\end{aligned}
$$

It is easy to see that

$$
\lim _{t \rightarrow \infty} S_{2}(t)=\frac{m_{1} Q}{b_{s} u_{2}+\mu} \quad \text { and } \quad \lim _{t \rightarrow \infty} S_{1}(t)=\left(1-\frac{m_{1} \mu}{b_{s} u_{2}+\mu}\right) \frac{Q}{\mu}
$$

Thus, for the reduced system, the DFE is globally asymptotically stable in $\Omega$.

To test for the second condition, $\mathrm{H} 2$, we compute $D_{Z} G(X, Z)=\frac{\partial G(X, Z)}{\partial\left(I_{1}, I_{2}, A\right)}$

$$
D_{Z} G\left(X^{*}, 0\right)=\left[\begin{array}{ccc}
\eta_{1}-k_{2} & p_{1} \eta_{1} b_{i} u_{2} & p_{2} \eta_{1} \\
\frac{p_{3} c \beta\left(1-u_{1}\right) \mu m_{1}}{k_{1}} & \frac{p_{3} c \beta\left(1-u_{1}\right) p_{1} \mu m_{1}}{k_{1}}-k_{3} & \frac{p_{3} c \beta\left(1-u_{1}\right) p_{2} \mu m_{1}}{k_{1}} \\
\delta\left(1-u_{3}\right) & \eta \delta\left(1-u_{3}\right) & -k_{4}
\end{array}\right]
$$


where $\eta_{1}=c \beta\left(1-u_{1}\right)\left(1-\frac{m_{1} \mu}{k_{1}}\right)$.

Using the relation in $\mathrm{H} 2$ above we have $\hat{G}(X, Z)=G(X, Z)-D_{Z} G\left(X^{*}, 0\right) Z$ and hence,

$$
\hat{G}(X, Z)=\lambda N\left(\begin{array}{c}
\frac{S_{10}}{N_{0}}-\frac{S_{1}}{N} \\
p_{3}\left(\frac{S_{20}}{N_{0}}-\frac{S_{2}}{N}\right) \\
0
\end{array}\right)
$$

From Theorem 3.1, it can be shown that $\hat{G}(X, Z) \geq 0$ and the following theorem is thus established.

Theorem 3.3. When $\mathcal{R}_{0} \leq 1$ in the absence of infective immigrants, the disease-free equilibrium $E_{0}$ is GAS.

Theorem 3.3 informs that immigration is a very important factor to consider when trying to combat the spread of HIV/AIDS and that the DFE when achieved can be maintained (after the condition $\mathcal{R}_{0}<1$ is met) only in the absence of immigration of infected individuals.

3.5. Existence of the Endemic Equilibrium. The model also exhibits an endemic equilibrium given by:

$$
\left\{\begin{aligned}
S_{1}^{*} & =\left[\frac{1-m_{1}-m_{2}-m_{3}}{\lambda^{*}+\mu}+\frac{b_{s} u_{2} m_{1}}{\left(p_{3} \lambda^{*}+k_{1}\right)\left(\lambda^{*}+\mu\right)}\right] Q, \\
S_{2}^{*} & =\frac{m_{1} Q}{p_{3} \lambda^{*}+k_{1}}, \\
I_{1}^{*} & =\frac{Q}{k_{2}}\left[m_{2}+\frac{b_{i} u_{2} m_{3}}{k_{3}}+\frac{\lambda^{*}}{\lambda^{*}+\mu}\left(\frac{b_{i} p_{3} u_{2} m_{1}\left(\lambda^{*}+\mu\right)}{\left(p_{3} \lambda^{*}+k_{1}\right) k_{3}}\right)\right]+\frac{\lambda^{*} S_{1}^{*}}{k_{2}} \\
I_{2}^{*} & =\left[\frac{m_{3}}{k_{3}}+\frac{m_{1} p_{3} \lambda^{*}}{k_{3}\left(p_{3} \lambda^{*}+k_{1}\right)}\right] Q, \text { and } \\
A^{*} & =\frac{\delta\left(1-u_{3}\right)}{k_{4}}\left(I_{1}^{*}+\eta I_{2}^{*}\right) .
\end{aligned}\right.
$$

Substituting the endemic equilibrium, (3.2) into the expression for $\lambda^{*}$ gives the following cubic equation.

$$
B_{3}\left(\lambda^{*}\right)^{3}+B_{2}\left(\lambda^{*}\right)^{2}+B_{1} \lambda^{*}+B_{0}=0
$$

where

$$
\begin{aligned}
\mathcal{B}_{0}= & \beta c k_{1} \mu^{2}\left\{k_{3}\left(\delta\left(1-u_{3}\right) p_{2}+k_{4}\right) m_{2}+\left[p_{2} \delta\left(1-u_{3}\right)\left(k_{2} \eta+b_{i} u_{2}\right)+k_{4}\left(p_{1} k_{2}+b_{i} u_{2}\right)\right] m_{3}\right\} \\
\mathcal{B}_{1}= & \mu\left(\mathcal{R}_{0}-1\right) k_{3} k_{2} k_{4} k_{1}++\psi \delta \mu k_{1}\left(1-u_{3}\right)\left(k_{3} m_{2}+m_{3}\left(b_{i} u_{2}+\eta k_{2}\right)\right) \\
& +\beta c\left[k_{3}\left(m_{2} p_{3} \mu^{2}+k_{1} m_{3}\right)\left(p_{2} \delta\left(1-u_{3}\right)+k_{4}\right)-\left(p_{2} \delta\left(1-u_{3}\right) \eta+p_{1} k_{4}\right) k_{2}\left(p_{3} \mu+k_{1}\right) m_{3}\right] \\
\mathcal{B}_{2}= & \mu p_{3}\left[\left(p_{2}\left(1-u_{3}\right)(\eta-1) \mu \delta+k_{4}\left(p_{1} k_{2}+b_{i} u_{2}\right)-k_{3}\right)\left(m_{1}+m_{3}\right)+k_{3}\left(\delta\left(1-u_{3}\right) p_{2}+k_{4}\right)\right] c \beta \\
& +\left[\mu\left(p_{3}\left(k_{2} \eta+b_{i} u_{2}\right)-k_{3}\right) m_{1}+\mu k_{3} p_{3} m_{2}+\left(\mu p_{3}+k_{1}\right)\left(k_{2} \eta+b_{i} u_{2}\right) m_{3}+k_{3} k_{1}\left(1-m_{3}\right)\right] \psi \delta\left(1-u_{3}\right) \\
& -k_{3} k_{2} k_{4}\left(\mu p_{3}+k_{1}\right) \\
\mathcal{B}_{3}= & p_{3}\left[\psi \delta\left(1-u_{3}\right)\left[\mu\left(m_{3}+m_{1}\right)(\eta-1)+k_{3}\right]-k_{3} k_{2} k_{4}\right]
\end{aligned}
$$


Clearly, $\mathcal{B}_{0}>0$ and hence by the Descarte's rule of signs, the nature of roots of equation (3.3) depend on the signs of $\mathcal{B}_{1}, \mathcal{B}_{2}$ and $\mathcal{B}_{3}$. The number of positive zeros of (3.3) is summarized in the Table 3.

The following theorem is therefore established from Table 3.

Table 3. Number of Possible Positive Roots of Endemic Polynomial in (3.3)

\begin{tabular}{|c|c|c|c|c|c|}
\hline Case & $\mathcal{B}_{0}$ & $\mathcal{B}_{1}$ & $\mathcal{B}_{2}$ & $\mathcal{B}_{3}$ & Number of positive roots \\
\hline 1 & + & + & + & + & 0 \\
\hline 2 & + & + & + & - & 1 \\
\hline 3 & + & + & - & + & 0,2 \\
\hline 4 & + & + & - & - & 1 \\
\hline 5 & + & - & + & + & 0,2 \\
\hline 6 & + & - & + & - & 1,3 \\
\hline 7 & + & - & - & + & 0,2 \\
\hline 8 & + & - & - & - & 1 \\
\hline
\end{tabular}

Theorem 3.4. From Table 3, the model:

i) Has no endemic equilibrium for case 1.

ii) Has a unique endemic equilibrium for cases 2, 4 and 8.

iii) Could have multiple endemic equilibria for cases 3, 5, 6 and 7.

Item, iii) above suggest the possibility of the backward bifurcation phenomenon, where for $\mathcal{R}_{0}<1$, there exists a stable DFE and stable endemic equilibrium. This phenomenon has been observed in several other models (See [17] and the references therein). This is further explored in section 4 . The presence of backward bifurcation implies that the classical condition that $\mathcal{R}_{0}$ be less than unity for disease eradication even though is necessary, might not be sufficient. Hence for effective disease control, other conditions besides $\mathcal{R}_{0}<1$ will have to be met.

For the special case when there are no infected immigrants (ie. $m_{2}=m_{3}=0$ ), equation (3.3) reduces to:

$$
\left[\mathcal{B}_{3}^{1}\left(\lambda^{*}\right)^{2}+\mathcal{B}_{2}^{1} \lambda^{*}+\mathcal{B}_{1}^{1}\right] \lambda^{*}=0
$$


where

$$
\begin{aligned}
\mathcal{B}_{1}^{1}= & \mu k_{1} k_{2} k_{3} k_{4}\left(\mathcal{R}_{0}-1\right) \\
\mathcal{B}_{2}^{1}= & \beta c \mu p_{3}\left\{\left[p_{2}\left(1-u_{3}\right)(\eta-1) \mu \delta+k_{4}\left(-k_{3}+p_{1} k_{2}+b_{i} u_{2}\right)\right] m_{1}+k_{3}\left(\delta\left(1-u_{3}\right) p_{2}+k_{4}\right)\right\} \\
& +\left[\mu\left(-k_{3}+p_{3}\left(k_{2} \eta+b_{i} u_{2}\right)\right) m_{1}+k_{3} k_{1}\right] \psi \delta\left(1-u_{3}\right)-k_{3} k_{2} k_{4}\left(\mu p_{3}+k_{1}\right) \text { and } \\
\mathcal{B}_{3}^{1}= & p_{3} k_{3}\left[\psi \delta\left(1-u_{3}\right)-k_{2} k_{4}\right]<0
\end{aligned}
$$

We note that when $\mathcal{R}_{0}>1$ then $\mathcal{B}_{1}^{1}>0$ and hence equation (3.4) will have a unique positive endemic equilibrium. The following result therefore follows:

Theorem 3.5. In the absence of infective immigrants:

i) The model has a unique endemic equilibrium for $\mathcal{R}_{0}>1$.

ii) The model has no endemic equilibrium when $\mathcal{B}_{2}^{1}<0$ and there is a possibility of backward bifurcation when $\mathcal{B}_{2}^{0}>0$ for $\mathcal{R}_{0}<1$.

\section{Bifurcation Analysis}

In this section, we make use of the center manifold theory [5] (as described in [7]) to study the bifurcation of the model. To do this, we make the change of variables; $x_{1}=S_{1}, x_{2}=S_{2}$, $x_{3}=I_{1}, x_{4}=I_{2}$ and $x_{5}=A$, so that the model is transformed into the following set of differential equations.

$$
\left\{\begin{aligned}
\frac{\mathrm{d} x_{1}}{\mathrm{~d} t} & =\left(1-m_{1}-m_{2}-m_{3}\right) Q+b_{s} u_{2} x_{2}-\left(\frac{c \beta\left(1-u_{1}\right)\left(x_{3}+p_{1} x_{4}+p_{2} x_{5}\right)}{x_{1}+x_{2}+x_{3}+x_{4}+x_{5}}+\mu\right) x_{1} \\
\frac{\mathrm{d} x_{2}}{\mathrm{~d} t} & =m_{1} Q-\left(\frac{p_{3} c \beta\left(1-u_{1}\right)\left(x_{3}+p_{1} x_{4}+p_{2} x_{5}\right)}{x_{1}+x_{2}+x_{3}+x_{4}+x_{5}}+b_{s} u_{2}+\mu\right) x_{2} \\
\frac{\mathrm{d} x_{3}}{\mathrm{~d} t} & =m_{2} Q+\frac{c \beta\left(1-u_{1}\right)\left(x_{3}+p_{1} x_{4}+p_{2} x_{5}\right) x_{1}}{x_{1}+x_{2}+x_{3}+x_{4}+x_{5}}+b_{i} u_{2} x_{4}-\left(\delta\left(1-u_{3}\right)+\mu\right) x_{3} \\
\frac{\mathrm{d} x_{4}}{\mathrm{~d} t} & =m_{3} Q+\frac{p_{3} c \beta\left(1-u_{1}\right)\left(x_{3}+p_{1} x_{4}+p_{2} x_{5}\right) x_{2}}{x_{1}+x_{2}+x_{3}+x_{4}+x_{5}}-\left(b_{i} u_{2}+\eta \delta\left(1-u_{3}\right)+\mu\right) x_{4} \\
\frac{\mathrm{d} x_{5}}{\mathrm{~d} t} & =\delta\left(1-u_{3}\right)\left(x_{3}+\eta x_{4}\right)-(\psi+\mu) x_{5}
\end{aligned}\right.
$$

Choosing $\beta=\beta_{*}$ as a bifurcation parameter (where $\beta_{*}$ is found from $\mathcal{R}_{0}=1$ ), it can be shown that the transformed equation (4.1) has a hyperbolic equilibrium point. Hence the center manifold theory ( Particularly, theorem 2.4) [5] can be used to analyze the model near $\beta=\beta_{*}$. The Jacobian of the transformed model can be shown to have left and right eigenvectors (corresponding to a simple eigenvalue) given respectively by

$$
v=\left[\begin{array}{lllll}
1 & p_{2} & 0 & -p_{2} & p_{1}
\end{array}\right]^{T} \text { and } w=\left[\begin{array}{lllll}
1 & 1 & 1 & 1 & 0
\end{array}\right]
$$

Using Theorem 2.4 of [5], the bifurcation coefficients are thus given by 


$$
\mathbf{a}=\frac{2 \Delta \beta_{*} c \mu\left(1-u_{1}\right)\left(1+p_{1}\right)}{\left(b_{s} u_{2}+\mu\right) Q} \text { and } \mathbf{b}=-c\left(1-u_{1}\right)\left[1+\frac{m_{1} \mu\left(2 p_{2} p_{3}-1\right)}{b_{s} u_{2}+\mu}\right]\left(1+p_{1}\right) .
$$

where $\Delta=\left(3-2 p_{2} p_{3}\right)\left(b_{s} u_{2}+\mu\right)+4 m_{1} \mu\left(2 p_{2} p_{3}-1\right)$.

Clearly, $\mathbf{b}<0$ and the sign of a depends on the sign of $\Delta$.

From Theorem 2 of Castillo-Chavez et al. [6], the following result is established .

Theorem 4.1. When $\mathcal{R}_{0} \leq 1$, the model (2.2) has an unstable endemic equilibrium and:

i. A locally asymptotically stable DFE for $\Delta<0$

ii. A stable DFE for $\Delta>0$

Theorem 4.1 above implies that the condition $\mathcal{R}_{0}<1$ does not guarantee disease eradication.

\section{Optimal Control Analysis}

In this section, we consider the controls $u_{1}, u_{2}$ and $u_{3}$ to be time-dependent. An optimal control problem can then be formed by seeking to minimize the following objective functional subject to the model equations in (2.2).

$$
\mathcal{J}=\int_{0}^{T}\left(\xi_{1} I_{1}+\xi_{2} I_{2}+\omega_{1} u_{1}^{2}+\omega_{2} u_{2}^{2}+\omega_{3} u_{3}^{2}\right) \mathrm{d} t
$$

In equations (5.1) above, $\xi_{1}, \xi_{2}, \omega_{1}, \omega_{2}$ and $\omega_{3}$ are positive weights associated with $I_{1}, I_{2}, u_{1}, u_{2}$ and $u_{3}$ respectively. the expressions $\omega_{i} u_{i}^{2}$ represent the unit cost associated with the control variable $u_{i}$. The objective functional $\mathcal{J}$ is chosen win line with related literature [2, and references therein]. Our interest then is to seek an optimal control triple, $\left(u_{1}^{*}, u_{2}^{*}, u_{3}^{*}\right)$ such that

$$
\mathcal{J}\left(u_{1}^{*}, u_{2}^{*}, u_{3}^{*}\right)=\min \left\{\mathcal{J}\left(u_{1}, u_{2}, u_{3}\right) \text { such that }\left(u_{1}, u_{2}, u_{3}\right) \in \mathcal{U}\right\}
$$

where

$\mathcal{U}=\left\{\mathcal{J}\left(u_{1}, u_{2}, u_{3}\right) \mid u_{i}\right.$ is lebseque measurable on $\left.[0, T] ; 0 \leq u_{i} \leq 1 \forall i=1,2,3\right\}$ is the set of admissible controls. Using the Pontryagin's Maximum Principle (PMP) [16], which provides necessary conditions for optimality, the optimal control problem (consisting of equation (2.2) and (5.1) ) can be converted into a problem of minimizing a Hamiltonian given by:

$$
\mathcal{H}=\frac{\mathrm{d} \mathcal{J}}{\mathrm{d} t}+\lambda_{1} \frac{\mathrm{d} S_{1}}{\mathrm{~d} t}+\lambda_{2} \frac{\mathrm{d} S_{2}}{\mathrm{~d} t}+\lambda_{3} \frac{\mathrm{d} I_{1}}{\mathrm{~d} t}+\lambda_{4} \frac{\mathrm{d} I_{2}}{\mathrm{~d} t}+\lambda_{5} \frac{\mathrm{d} A}{\mathrm{~d} t}
$$

The following thereom is thus obtained. 
Theorem 5.1. Let $\left(u_{1}^{*}, u_{2}^{*}, u_{3}^{*}\right)$ be an optimal control triple with $\left(S_{1}^{*}, S_{2}^{*}, I_{1}^{*}, I_{2}^{*}, A^{*}\right)$ being the corresponding solution to the state equations (2.2). Then there exists adjoint variables $\lambda_{i} \forall i=1,2 \ldots 5$ such that

$\frac{\mathrm{d} \lambda_{1}}{\mathrm{~d} t}=-\frac{\partial \mathcal{H}}{\partial S_{1}}, \frac{\mathrm{d} \lambda_{2}}{\mathrm{~d} t}=-\frac{\partial \mathcal{H}}{\partial S_{2}}, \frac{\mathrm{d} \lambda_{3}}{\mathrm{~d} t}=-\frac{\partial \mathcal{H}}{\partial I_{1}}, \frac{\mathrm{d} \lambda_{4}}{\mathrm{~d} t}=-\frac{\partial \mathcal{H}}{\partial I_{2}}$ and $\frac{\mathrm{d} \lambda_{5}}{\mathrm{~d} t}=-\frac{\partial \mathcal{H}}{\partial A}$

The adjoint (or Co-state) equations are thus given by:

$$
\left\{\begin{array}{l}
\frac{\mathrm{d} \lambda_{1}}{\mathrm{~d} t}=\frac{\Lambda \lambda}{N}+p_{3}\left(\lambda_{2}-\lambda_{4}\right) \lambda+\left(\lambda_{2}-\lambda_{1}\right) b_{s} u_{2}+\lambda_{2} \mu \\
\frac{\mathrm{d} \lambda_{2}}{\mathrm{~d} t}=\frac{\Lambda \lambda}{N}+\left(\lambda_{1}-\lambda_{3}\right) \lambda+\lambda_{1} \mu \\
\frac{\mathrm{d} \lambda_{3}}{\mathrm{~d} t}=-\xi_{1}-\Lambda\left(\frac{c \beta\left(1-u_{1}\right)}{N}-\frac{\lambda}{N}\right)+\left(\lambda_{3}-\lambda_{5}\right) \delta\left(1-u_{3}\right)+\lambda_{3} \mu \\
\frac{\mathrm{d} \lambda_{4}}{\mathrm{~d} t}=-\xi_{2}-\Lambda\left(\frac{c \beta\left(1-u_{1}\right) p_{1}}{N}-\frac{\lambda}{N}\right)+\left(\lambda_{4}-\lambda_{3}\right) b_{i} u_{2}+\left(\lambda_{4}-\lambda_{5}\right) \eta \delta\left(1-u_{3}\right)+\lambda_{4} \mu \\
\frac{\mathrm{d} \lambda_{5}}{\mathrm{~d} t}=-\Lambda\left(\frac{c \beta\left(1-u_{1}\right) p_{2}}{N}-\frac{\lambda}{N}\right)+\lambda_{5}(\psi+\mu) \\
\lambda_{1}(T)=0, \lambda_{2}(T)=0, \lambda_{3}(T)=0, \lambda_{4}(T)=0, \lambda_{5}(T)=0
\end{array}\right.
$$

where $\Lambda=\left[\left(\lambda_{3}-\lambda_{1}\right) S_{1}+p_{3}\left(\lambda_{4}-\lambda_{2}\right) S_{2}\right]$.

The optimal control triple is found by solving optimality equations, $\frac{\partial H}{\partial u_{i}}=0$, so that we have the following characterizations;

$$
\left\{\begin{array}{l}
u_{1}^{*}=\max \left\{0, \min \left\{1, \frac{c \beta\left(I_{1}+p_{1} I_{2}+p_{2} A\right) \Lambda}{2 N \omega_{1}}\right\}\right\}, \\
u_{2}^{*}=\max \left\{0, \min \left\{1, \frac{\left(\lambda_{4}-\lambda_{3}\right) b_{i} I_{2}+\left(\lambda_{2}-\lambda_{1}\right) b_{s} S_{2}}{2 \omega_{2}}\right\}\right\} \\
u_{3}^{*}=\max \left\{0, \min \left\{1, \frac{\delta\left[\left(\lambda_{5}-\lambda_{3}\right) I_{1}+\left(\lambda_{5}-\lambda_{4}\right) \eta I_{2}\right]}{2 \omega_{3}}\right\}\right\} .
\end{array}\right.
$$

\section{Numerical Simulation and Discussion of Results}

In this section, the model (2.2) and the resulting optimal control problem are numerically solved to examine the impact of implementation of the controls. In order to determine the impact of implementing various combination of the controls, the following combinations are implemented each at a time:

Strategy A: Implementing Condom use and behavioral change control (ie. $u_{1} \neq 0$ and $u_{2} \neq$ $\left.0 u_{3}=0\right)$.

Strategy B: Implementing Condom use and anti-retro-viral therapy (ie. $u_{1} \neq 0$ and $u_{2}=$ $\left.0 u_{3} \neq 0\right)$.

Strategy C: Implementing behavioral change control and anti-retro-viral therapy (ie. $u_{1}=0$ and $\left.u_{2} \neq 0 u_{3} \neq 0\right)$.

Strategy D: Implementing all controls (ie. $u_{1} \neq 0, u_{2} \neq 0$ and $u_{3} \neq 0$ ). 
Using the parameter values in Table 1, we solve the basic model (2.2) without controls and also the optimal control problem for of the strategies above. For each strategy, we calculate the total number of infectives during the implementation and called it "Total Infections" nd the cost of implementation. The total number of infections prevented as a result of the implementation of the strategy is calculated by the taking the difference between the Total Infections for the strategy and the total Infections for the basic model without controls. These are presented in Table 4.

TABLE 4. Total Infections averted and Total Cost of Intervention Strategies.

\begin{tabular}{|ccl|}
\hline STRATEGY & TOTAL INFECTIONS AVERTED & COST $(\$)$ \\
\hline Uncontrolled & 0 & 0 \\
C & -3233613 & 89.8153 \\
A & 498479 & 10.2192 \\
D & 498752 & 90.0599 \\
B & 498761 & 80.0799 \\
\hline
\end{tabular}

In order to determine which of the strategies is most cost-effective, we employ the IncrementalCost-Effectiveness ratio (ICER), which is often used to compare two intervention schemes. It is generally defined as the additional cost per additional health outcome of an intervention. The ICER between two interventions, $A$ and $B$ is defined as follows:

$\operatorname{ICER}(\mathrm{B} / \mathrm{A})=\frac{\text { Cost of B-Cost of A }}{\text { Infections Averted by B-Infections Averted by A }}$.

Strategy $\mathrm{C}$ is clearly observed not to produce desired results in terms of averting infections. So, we exclude Strategy C from the list of possible cost-effective strategies, leaving A, B and D. Next, we compare strategy $A$ and $D$ by calculating incremental-cost-effectiveness ratio of $A$ and $\mathrm{D}$ as follows:

$\operatorname{ICER}(\mathrm{A})=2.0501 \mathrm{E}-05, \operatorname{ICER}(D \mid A)=\frac{90.0599-10.2192}{498752-498479}=0.2924$

We observe that Strategy D has a higher ICER than A which implies that strategy D is more costly and hence dominated by strategy A. Therefore, we exclude strategy $\mathrm{D}$ in the list of possible cost-effective strategies. Next, we compare strategies A and B by calculating the ICER of $\mathrm{B}$ as follows:

$\operatorname{ICER}(\mathrm{A})=2.0501 \mathrm{E}-05, \operatorname{ICER}(A \mid B)=\frac{80.0799-10.2192}{498761-498479}=0.2477$.

We observe that Strategy B has a higher ICER than A which implies that strategy B is more 
costly and hence dominated by strategy A. We conclude that strategy $\mathrm{A}$ is the most costeffective strategy than can be employed in the effort to combat the spread of the disease. The graphs of numerical results for the best strategy are presented in Figure 1.
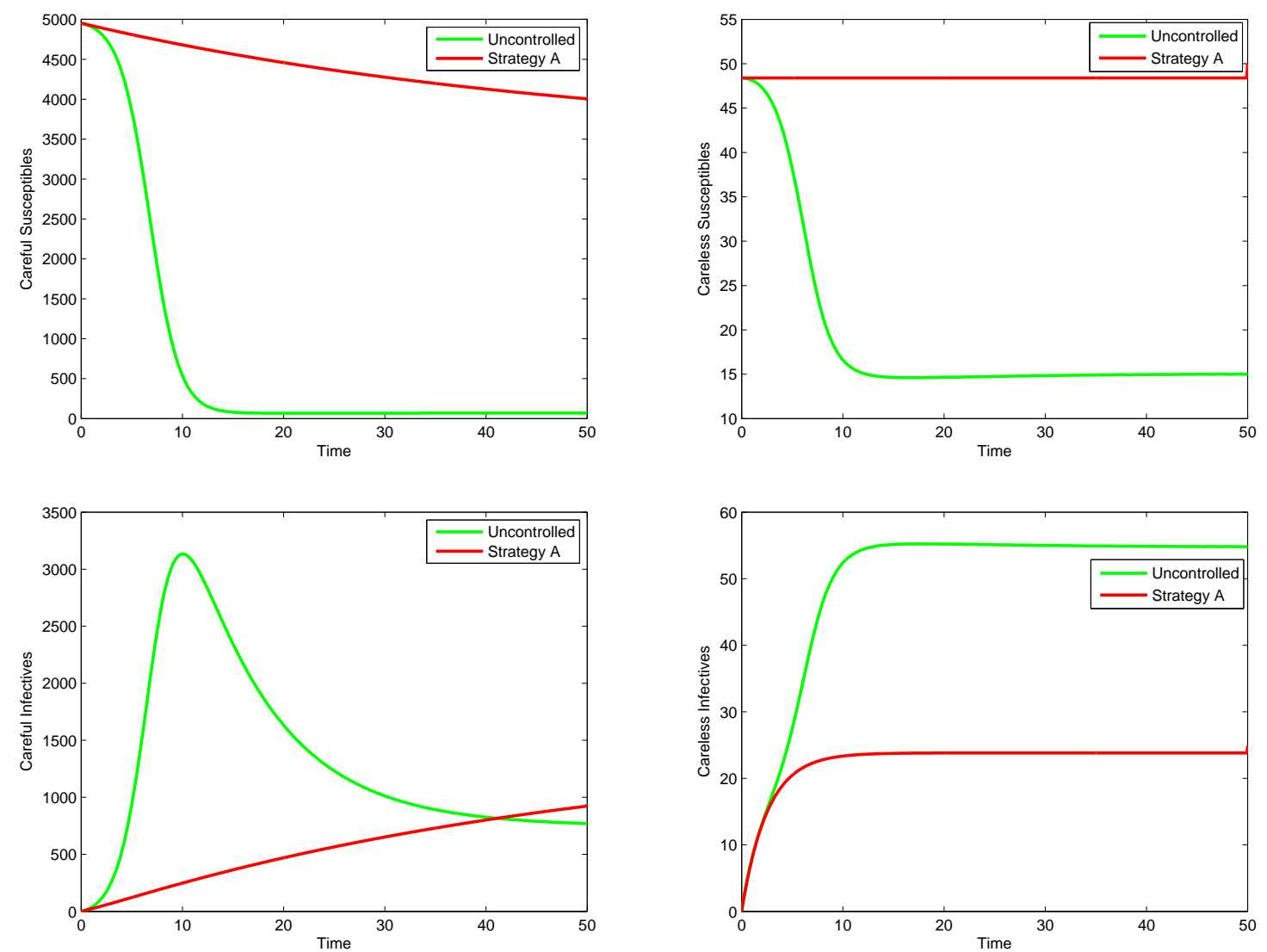

Figure 1. Simulation of basic Model (2.2) and Resulting Optimal Control Problem for the Most Cost-Effective Strategy.

\section{Conclusion}

In this paper, a deterministic model is proposed to study the dynamics of HIV/AIDS in the presence of careful and careless Susceptibles and Infectives. Basic properties of the model are presented. The mode is extended into an optimal control problem which is qualitatively analysed and numerically solved for various combinations of intervention strategies. Incremental-cost-effectiveness ratio analysis showed that the best strategy that should be employed in the fight against HIV/AIDS is one that involves condom use (Prevention) and behavioural change towards sex. 


\section{REFERENCES}

[1] T. Agraj, R. Naresh, S. Dillep, Modeling the effects of screening of unaware infectives on the spread of HIV infection, Appl. Math. Comput. 184 (2007), 1053-1068.

[2] S. Baba, O. D. Makinde, I. Y. Seini, Mathematical analysis of the effects of HIV-Malaria Co-infection on workplace productivity, Acta Biotheoretica, 63(2) (2015), 151-182.

[3] J. T. Bertrand, K. O'Reilly, J. Denison, R. Anhang, M. Sweat, Systematic review of the effectiveness of mass communication programs to change HIV/AIDS-related behaviors in developing countries, Health Educ. Res. 21(4) (2006), 567-597.

[4] Fred Brauer, P. van den Driessche, Models for transmission of disease with immigration of infectives, Math. Biosci. 171(2) (2001), $143-154$.

[5] J. Carr, Applications Center manifold Theory, Springer Velag, New York, 1981.

[6] C. Castillo-Chavez, S. Blower, P. Driessche, D. Kirschner, A. A. Yakubu, Mathematical approaches for emerging and reemerging infectious diseases: models, methods, and theory, Springer, 2002.

[7] C. Castillo-Chavez, B. Song, Dynamical models of tuberculosis and their applications, Math. Biosci. Eng, $1(2)(2004), 361-404$.

[8] R. V. Culshaw, S. Ruan, A delay-differential equation model of HIV infection of $\mathrm{CD}_{4}+\mathrm{T}$ cells, Math. biosci. 165(1) (2000), 27-39.

[9] M. I. Daabo, S. Baba, Modelling the effect of irresponsible infective immigrants on the transmission dynamics of HIV/AIDS., Adv. Appl. Math. Biosci. 3 (2012), 31-40.

[10] P. van den Driessche, J. Watmough, Reproduction numbers and sub-threshold endemic equilibria for compartmental models of disease transmission, Math. Biosci. 180 (2002), 29-48.

[11] P. Essunger, A. S. Perelson, Modeling HIV infection of $\mathrm{CD}_{4}+\mathrm{T}$-cell subpopulations, J. Theor. Biol. 170(4) (1994), 367-391.

[12] D. Finzi, J. Blankson, J. D. Siliciano, et al., Latent infection of $\mathrm{CD}_{4}+\mathrm{T}$ cells provides a mechanism for lifelong persistence of HIV-1, even in patients on effective combination therapy, Nature Med. 5(5) (1999), 512-517.

[13] A. Gumel, P. N. Shivakumar, B. M. Sahai, A mathematical model for the dynamics of HIV-1 during the typical course of infection, Nonlinear Anal.: Theory Meth. Appl. 47(3) (2001), 1773-1783.

[14] H. W. Hethcote, The mathematics of infectious diseases, SIAM Rev. 42(4) (2000), 599-653.

[15] Y. H. Hsieh, C. H. Chen, Modeling the social dynamics of a sex industry: its implications for spread of HIV/AIDS, Bull. Math. Biol., 66 (2004), 143-166.

[16] L. S. Pontryagin, The mathematical theory of optimal processes, Volume 4, CRC Press, 1962.

[17] O. Y. Sharomi, Mathematical Analysis of Dynamics of Chlamydia trachomatis, Doctoral Thesis, University of Manitoba, 2010. 